\title{
The effect of syntactical violation upon the recall of form class
}

\section{DENNIS L. ANDERSON AND JOE L. BYERS, DEPARTMENT OF EDUCATIONAL PSYCHOLOGY, MICHIGAN STATE UNIVERSITY, East Lansing, Mich. 48823}

The recall of form class was investigated by having Ss learn word lists as sentences and anagrams. Although nouns had the highest recall and adverbs the lowest for both types of word lists, differences between adjacent form classes at the point of transition from the noun phrase to the verb phrase decreased with practice for sentences but not for anagrams. Recall of form class was found to depend to some extent upon the verbal context of the word list.

Studies by Davidson (1966) and Simpson (1965) suggest that the recall of various form classes may be a function of the degree of syntactical violation present in the list in which words are learned and the frequency with which the $S$ has been exposed to the list. Using anagrams, as a form of syntactical violation, and syntactically correct sentences in a free recall task, this study sought to determine if form classes were recalled differently because of form class itself or because of an interaction between form class and the context in which a particular word was learned. It was hypothesized that recall would be significantly higher for all form classes learned within the context of a sentence due to the facilitating effects of syntactical relationships between words.

A study by Johnson (1965) indicates that the probability of a word-to-word transitional error is greater for transitions occurring between phrases than for transitions occurring within phrases. In terms of the present study, this means that the greatest differences in recall between two adjacent words would be found at the end of the noun phrase and the beginning of the verb phrase, which in this case would consist of the first noun and the adverb. For sentences, it was hypothesized that this difference would decrease across trials due to the effects of syntactical relationships and serial position. If words within the noun phrase and verb phrase are recalled early in practice as functional units, then the difference in recall between the first noun and the adverb should show a significant decrease across trials, eventually resulting in the entire sentence being recalled as a single functional unit. For anagrams, this decrease in differences across trials would depend mainly upon the Ss being able to recognize the list of words.

Method. The Ss consisted of 49 fifth-grade students in a midwestern elementary school. In a replication of the study in two other schools the same results were found. Therefore they will not be reported here.

Eight six-word sentences were constructed from 48 different high frequency words according to the following syntactical structure: Adjective $\left(A_{1}\right)$ - Noun $\left(N_{1}\right)$ - Adverb (Adv) - Verb (V) - Adjective $\left(A_{2}\right)$ - Noun $\left(N_{2}\right)$.

The following procedure was used to create the two types of word lists. Four sentences were randomly selected from the basic set of eight and presented intact, constituting sentence list (e.g., strong boys usually build large boats). The remaining four sentences were scrambled, thus creating four sentence anagrams (e.g., ideas new find children little always).

In order to establish greater generality three modes of presentation were used: aural, visual, and aural-visual. The Ss were randomly assigned to the three presentation modes in groups containing 16, 16, and 17 Ss. They were tested as intact groups in which each list was presented to the group six times, once in each block of trials. A trial consisted of a list of 24 words in four six-word strings. The lists were presented at a 1-sec pace with a 2 -sec pause between each six-word string. Following the presentation the Ss were given a 2-min recall period in which to write the stimulus words. A 1-min rest period was given after each block of two trials. To prevent confounding of list type with practice effects the order of list presentation within blocks of trials was systematically varied so that each list appeared equally often in the first and second position. To reduce possible serial effects with in the sentence and anagram list types, the order of presentation within each of the four strings within a list type was randomly varied across the six trials.
A response was scored correct if the word appeared anywhere within the 24 words to which the $S$ had just been exposed. Form class scoring for anagrams was determined by the position of a word in the original sentence prior to being scrambled. A factorial analysis of variance procedure and the Scheffe method of post hoc analysis were used to analyze these data.

Results and Discussion. Mode of presentation had no main effect or interaction with form class, list type, and trials. Therefore the following presentation of results will not consider this variable.

Significant main effects were found for form class $(F=49.23$, $\mathrm{df}=2 / 240, \mathrm{p}<.0005)$, list type $(\mathrm{F}=690.75, \mathrm{df}=1 / 48, \mathrm{p}<$ $.0005)$, and trials $(F=73.00, d f=5 / 240, p<.0005)$. However, of primary concern is the significant interaction of these three variables $(F=2.56, \mathrm{df}=25 / 1200, \mathrm{p}<.0005)$, since it provides a means of observing the operation of syntactical constraint upon the recall of words of various form classes when they are learned within the different types of word lists. The word lists can be considered as two types of learning tasks: sentences, which have within them a certain amount of syntactical order; and anagrams, which have no order but are capable of having a syntactical order imposed upon them by the $S$.

From Table 1 it can be seen that for both sentences and anagrams the highest recall was obtained for the first noun and the lowest recall for the adverb in a comparison of mean recall scores for all trials combined. For sentences, the lower mean recall score for adverbs might be explained by the presence of a serial position effect, whereas for anagrams, this would not be the case since the adverbs were randomly distributed throughout the list. Adverbs were still recalled less well than other form classes, suggesting that adverbs may be a more difficult form class to recall in a learning task.

Pairwise contrasts between adjacent words in sentences and adjacent words in reconstructed sentences resulting from the solution of anagrams suggest that words within phrases may be more dependent upon each other in recall than words between phrases. Differences among adjacent words within phrases do not appear after the first trial for sentences, which may indicate that words within the phrases facilitate the recall of each other when they are learned in a meaningful syntactical relationship.

From Fig. 1 it can be seen that for sentences, differences in recall for the two adjacent words at the point of transition from

Table 1

Mean Recall for Form Class Within List Types and Trials

\begin{tabular}{|c|c|c|c|c|c|c|c|c|}
\hline Trial & & $\mathbf{A}_{1}$ & $\mathrm{~N}_{1}$ & Adv. & V & $\mathbf{A}_{2}$ & $\mathbf{N}_{2}$ & $\begin{array}{r}\text { Trial } \\
\text { Means }\end{array}$ \\
\hline \multirow{2}{*}{1} & $\mathbf{S}$ & 2.7 & 3.2 & 1.9 & 2.4 & 2.0 & 2.4 & 2.4 \\
\hline & $\boldsymbol{A}$ & .8 & 1.4 & .6 & .9 & 1.1 & .9 & .9 \\
\hline \multirow{2}{*}{2} & $\mathbf{S}$ & 3.2 & 3.4 & 2.5 & 2.8 & 2.9 & 3.1 & 3.0 \\
\hline & A & 1.1 & 2.0 & 1.4 & 1.3 & 1.8 & 1.9 & 1.6 \\
\hline \multirow{2}{*}{3} & S & 3.4 & 3.5 & 3.0 & 3.2 & 3.1 & 3.4 & 3.3 \\
\hline & A & 1.4 & 1.9 & 1.2 & 1.3 & 1.6 & 1.8 & 1.5 \\
\hline \multirow{2}{*}{4} & $\mathbf{S}$ & 3.5 & 3.5 & 3.0 & 3.3 & 3.3 & 3.4 & 3.3 \\
\hline & A & 1.5 & 2.2 & 1.4 & 1.4 & 1.8 & 2.0 & 1.7 \\
\hline \multirow{2}{*}{5} & $S$ & 3.7 & 3.7 & 3.4 & 3.5 & 3.4 & 3.6 & 3.5 \\
\hline & $\mathbf{A}$ & 1.8 & 2.4 & 1.8 & 1.8 & 2.0 & 2.2 & 2.0 \\
\hline \multirow{2}{*}{6} & $\mathbf{S}$ & 3.8 & 3.8 & 3.5 & 3.6 & 3.5 & 3.6 & 3.6 \\
\hline & A & 1.7 & 2.2 & 1.4 & 1.7 & 2.0 & 2.0 & 1.8 \\
\hline Mean & & 2.4 & 2.8 & 2.1 & 2.3 & 2.4 & 2.6 & \\
\hline
\end{tabular}




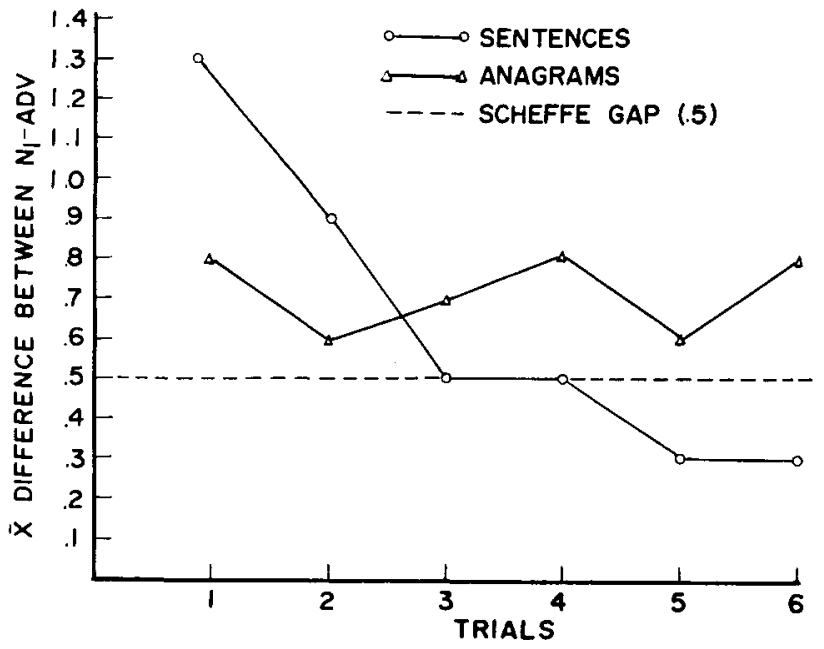

Fig. I. Mean difference across trials between the first noun and adverb for sentences and anagrams.

the noun phrase to the verb phrase (differences between the first noun and the adverb) decrease throughout all six trials, exceeding the critical value in only the first two trials. This difference exceeds the Scheffe critical value at each trial for anagrams. The pattern of recall for sentences might be explained by the fact that a high percentage of $S s$ were reaching a ceiling level in noun recall by the third trial. Adverbs continued to be recalled better in the last three trials, thus decreasing the differences between nouns and adverbs. However, a relatively stable SD for each trial indicates that such a restriction in range does not seem to be present.

The way in which the S learns the list of words may also explain the pattern of sentence recall. Syntactical relationships among words may facilitate recall over the first three trials, whereas in later trials the $\mathrm{S}$ may learn the words as a serial list, making use of associations between words in order to eventually recall the entire list as a functional unit. The association between the first noun and adverb would also be strengthened, causing the difference in recall to decrease in later trials. For anagrams, the pattern of differences between the first noun and the adverb may have resulted from the Ss using two competing strategies. Some Ss may have attempted to put the noun and adverb together in reorganizing the sentence. Others may have tried to learn the words as a serial list, which would result in the fitst noun and adverb being adjacent words only by chance. With Ss using either strategy it seems reasonable to expect that differences in recall would not show the continual decrease with practice, as was found to be the case with sentences.

Differences in recall may be due in part to form class itself. However, these differences seemed to depend, to a greater extent, upon the type of verbal context in which the words were learned.

\section{REFERENCES}

DAVIDSON, R. E. Semi-grammaticalness in the free learning of sentences. Unpublished doctoral dissertation, University of California, Berkeley, 1966.

JOHNSON, N. F. The psychological reality of phrase-structure rules. J. verbal Learn, verbal Behav., 1965, 4, 469-475.

SIMPSON, W. E. Effects of approximation to sentence word-order and grammatical class upon the serial learning of word lists. J. verbal Learn. verbal Behav., 1965, 4, 510-514. 